\title{
Increased intrinsic stiffness and mineralization in femurs of adult rats after high intensity treadmill running training
}

\author{
Clarisa Bozzini $^{\text {a }}$, Ginés Viscor ${ }^{\mathrm{b}}$, Juan C. Ríos Kristjánsson ${ }^{\mathrm{b}}$, Emilio Picasso ${ }^{\mathrm{c}}$, \\ Graciela Champin ${ }^{\text {a }}$, Rosa M. Alippi ${ }^{a}$ and Carlos E. Bozzini ${ }^{\mathrm{a}, *}$ \\ ${ }^{a}$ Cátedra de Fisiología, Facultad de Odontología, Universidad de Buenos Aires, Argentina \\ E-mail: cebozi@fisio.odon.uba.ar \\ ${ }^{\mathrm{b}}$ Departament de Fisiología i Immunología, Universitat de Barcelona, España \\ ${ }^{\mathrm{c}}$ Facultad de Ingeniería, Universidad de Buenos Aires, Argentina
}

Received 24 October 2016

Accepted 1 June 2017

\begin{abstract}
.
BACKGROUND: Physical activity plays a tremendous role in determining bone mechanical behavior, which is superimposed to gravidity.

OBJECTIVE: Compare the geometric and material responses of the rat femur to a high intensity treadmill running training of a relatively short duration, as assessed by 3 -point mechanical test.

METHODS: Mature male rats $(180.0 \pm 30 \mathrm{~g})$ were assigned ( 7 rats/group) to no exercise (NE) or treadmill exercise (EX). After a preconditioning period, the running speed was set at $45 \mathrm{~cm} \cdot \mathrm{seg}^{-1}$ during $2 \mathrm{wks}$, frequency $5 \mathrm{~d} / \mathrm{wk}$, 2-hour sessions/day. Body weight and weight of the crural quadriceps were registered at euthanasia. The right femur was mechanically tested through 3-point bending. The left femur was ashed to estimate bone mineral content. Geometric and material bone properties were estimated directly or calculated by appropriate equations.

RESULTS: 1) Final body weight was $14 \%$ reduced in EX rats, while the crural quadriceps was $47 \%$ increased. Yield and fracture loads, and structural stiffness were significantly higher in the EX rats, as were the apparent elastic modulus, the bone mineral content and the degree of mineralization. Geometric properties were not affected.

CONCLUSIONS: High intensity treadmill running training increases bone strength and stiffness by increasing material stiffness and mineralization, without affecting geometric bone parameters.
\end{abstract}

Keywords: Bone biomechanics, treadmill exercise, rat femur, bone strength

\section{Introduction}

Mammalian bone and skeletal muscle involved with locomotion develop in close association from the somatic mesoderm and accumulate their final adult mass to specific genetic instructions and environmental cues [1]. Changes in muscle and bone mass brought about by exercise, disuse or aging are also tightly correlated in both human and experimental models.

\footnotetext{
${ }^{*}$ Corresponding author: Carlos E. Bozzini, Marcelo T. de Alvear 2142, Buenos Aires 1122, Argentina. Tel.: 5411-5287-6186; Fax: 5411-5287-6007; E-mail: cebozi@ fisio.odon.uba.ar.
} 
A fundamental determinant of peak bone and muscle mass is genetic background. Superimposed to these genetic determinants of bone and muscle mass are anabolic stimuli that occurs postnatally, the most dominant of which is puberty. During the pubertal growth spurt, bone and muscle mass accumulate rapidly under the influence of GH, IGF-1, and sex hormones [2]. In addition to the genetic determinants and humoral factors affecting musculoskeletal mass, the level of physical activity in which human or animals engages plays a tremendous role in determining postnatal muscle and bone mass, which is superimposed to the forces associated with gravidity [3-5].

The intrinsic relationship between muscle and bone is described by the mechanostat theory, which postulates that increasing maximal muscle force during growth or in response to increasing loading will positively affect bone mass, size and strength [6].

According to the mechanostat theory, mechanical factors are considered as the primary factors determining bone strength. Most of the mechanical forces that act on the skeleton during physical activities are generated either through impact with the ground (gravitational or ground-reaction forces) or through skeletal muscle phasic contractions (muscle or joint-reaction forces) [7].

The effect of treadmill exercise on bone mass in rodents has been previously studied [8-12]. The time and the degree of the endurance treadmill running training were not uniform in the different studies, although most of them revealed that this type of exercise has effects on bone density, size and shape, resulting in substantial improvements in mechanical strength. However, subnormal values of bone mass, size, and strength were also described in association with enhanced intrinsic bone strength [13] which may represent real benefits in the biomaterial quality of bone [12]. Increased Young's modulus and higher concentration of $\mathrm{Ca}$ in femurs of trained rats were also described [9]. In general, increases in bone mineral density (BMD) can be obtained by a moderate running load $(15 \mathrm{~m} / \mathrm{min}$ for $30 \mathrm{~min})$ at frequencies of 4 and 5 days per week [11]. It has also been reported that the effect of exercise on bone at an older age is different from that at other ages [14].

It has been previously reported [10] that when the rat, a tetrapedal animal, is running on a treadmill, there is a greater mechanical loading on the appendicular bones than on the axial bones. Namely, the tibia and femur receive much more mechanical loading than the lumbar spine during running on the treadmill, which explains the preferential use of both bones in experiments dealing with the effects of exercise on bone strength.

Bone can be studied at several levels of organization. The "whole-bone" quality, or structural properties, considers the entire bone as a structure, which incorporates the properties of the materials that compose the whole bone (which can be [Ca]-related or [Ca]-unrelated) as well as the geometric properties, or architectural design, which include the trabecular network and the cortical shell [15]. The whole-bone quality is essentially a function of two mechanical properties: stiffness (or resistance to deformation) and strength (or resistance to fracture).

The "mechanical bending test" of bone, performed in vitro, which provides direct measurement of the whole-bone structural properties (and in combination with geometrical data allows indirect calculations of some bone material properties) was applied in the present study to estimate the effects of treadmill running training on the femoral shafts of adult rats. The degree of the endurance treadmill running was more intense, although applied for a shorter time, than those usually used in most studies performed on the subject. It is conceivable that higher running speeds would induce higher ground reaction forces and thus the effects on bone biomechanics would be more marked or different. 


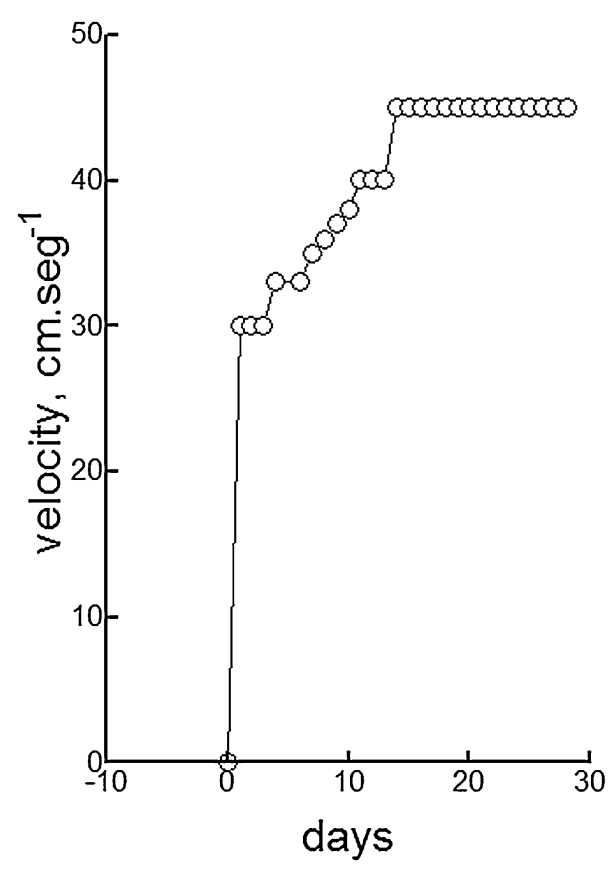

Fig. 1. Diagram of the training schedule.

\section{Materials and methods}

\subsection{Animal and experimental design}

Fourteen Sprague-Dawley male rats, 8 weeks old, weighing $180.0 \pm 30.0 \mathrm{~g}$ were used throughout. Young adult rats were chosen to study the effect of training still within the growth period but not as accelerated as in younger animals. Rats were kept in a room maintained at $22 \pm 2^{\circ} \mathrm{C}$ with a $12: 12 \mathrm{~h}$ light/dark cycle. Rats were provided free access to standard rat chow and water throughout the 4 wks study. Rats were randomly assigned ( $n=7$ rats/group) to no exercise (NE) or treadmill exercise (EX). The treadmill exercise regimen adopted corresponds to a high-intensity intermittent endurance protocol. It consisted of a preconditioning period in which running speed was gradually increased from $30 \mathrm{~cm} . \mathrm{seg}^{-1}$ to $45 \mathrm{~cm} . \mathrm{seg}^{-1}, 0 \%$ incline, during $2 \mathrm{wk}$, at a frequency of $5 \mathrm{~d} / \mathrm{wk}$, 2-hour sessions per day (morning and afternoon) (see Fig. 1). An equal long lasting period of exercise training was followed in which the running speed was maintained at $45 \mathrm{~cm} . \mathrm{seg}^{-1}$. An electric stimulus was turned on for less than $2 \mathrm{~s}$ when the animals stayed on the electric grill for longer than $10 \mathrm{~s}$.

Body weight of animals was recorded at the end of the experimental period. At euthanasia, the right crural quadriceps was dissected and weighed in a Mettler scale. We have chosen the femur for this experiment because it is subject to both gravity derived forces prevailing in static conditions, and movement derived forces prevailing in dynamic conditions. Additionally, the femur is highly adapted to biomechanical study due to its relatively large size, and its shape that resembles a beam. This shape is appropriate for the 3-point bending test and subsequent analysis. The right and left femurs were collected from each rat. The bones were defleshed with care being taken not to damage the periostium. The left femur was ashed. The right femur was weighed and stored at $-20^{\circ} \mathrm{C}$ wrapped in gauze soaked with Ringer's solution in sealed plastic bags [16] until analyzed. 


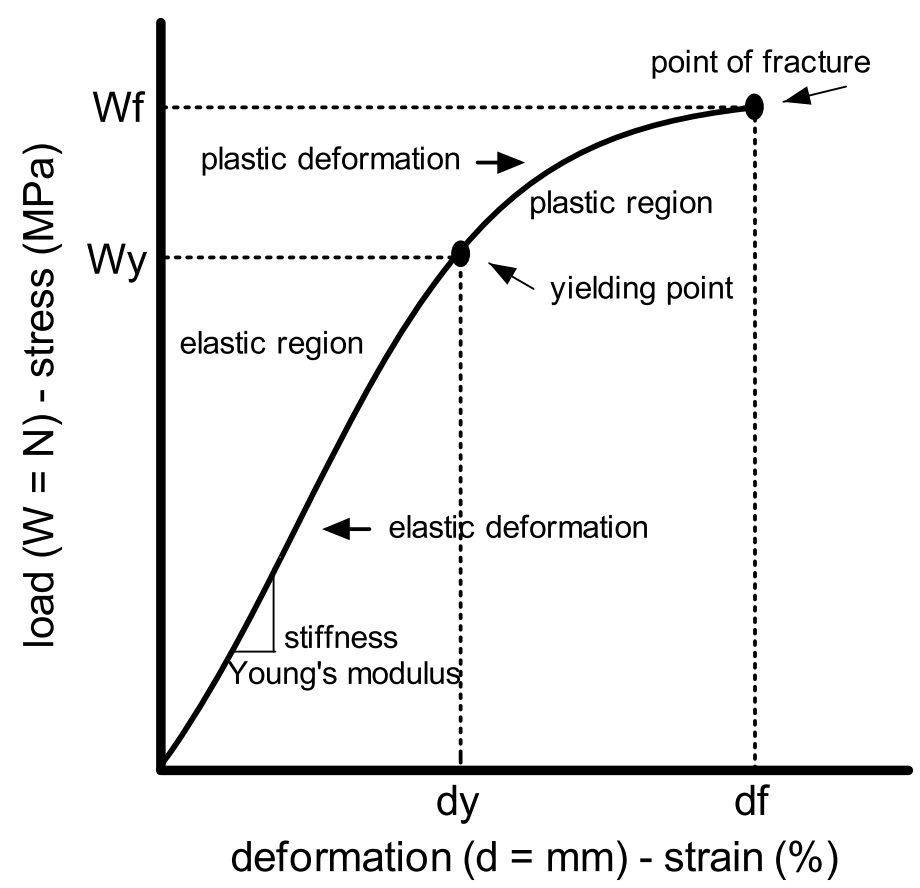

Fig. 2. The mechanical test generates a load/deformation curve from which several parameters can be measured. These parameters can be normalized after adjusting for the sample size (cross-sectional area or moment of inertia) allowing load conversion to stress and deformation to strain, and obtaining the "stress/strain" curve. The first linear portion of the curve is known as the "elastic region", where there is a proportional deformation (strain) with increased load (stress) exerted; when the load (stress) is removed, bone return to its original shape. After the "yielding point", increasing load (stress) causes permanent damage to the bone structure. The "point of fracture" corresponds to the maximum load (stress) the bone can sustain without breaking. The slope of the curve within the elastic region is a measure of the "stiffness" of the whole bone (extrinsic property) when obtained from the W/d curve. When obtained from the $S / S$ curve, it is called "Young's modulus" and is an index of the stiffness of the bone material (intrinsic property). Strength can be defined from the point of fracture or the point of yielding.

The experimental plan was approved by the Ethics Board of the Faculty of Odontology of the University of Buenos Aires.

\subsection{Biomechanical testing}

On the day of testing, each bone was thawed at room temperature before analysis. To assess cortical bone mechanical properties, the right femur was tested in 3-point bending [16] which combines compression and tension. Each bone was placed horizontally with the anterior side facing down on two transverse supports and central along its length. Load was applied perpendicularly to the long axis of the bone until fracture. The test machine (Instron model 4442, Instron Corp., Canton, MA, USA) was operated in stroke control at a constant rate of $5 \mathrm{~mm} / \mathrm{min}$ in order to obtain the load (W)/deformation (d) curves ( W/D curve, Fig. 2), showing both the elastic (linear) and the plastic phases separated by the yielding point (departure from linearity). "Elastic" means linear proportionality between the load and the reversible deformation. "Plastic" refers to nonlinear relationship between the load and the irreversible deformation [15]. The test enables graphic determination of the main structural mechanical properties of bone shafts as beams [16] which essentially measures the resistance to both deformation (stiffness) and fracture (strength). This way of load is useful for describing the "static properties" of the bone structure [15]. The structural properties are those corresponding to the whole bone as an organ and are derived 
from the W/d curve. They are: (i) load at the yielding point or elastic limit $\left(W_{y}\right)$, represents the end point of the elastic deformation of the bone (yielding point) and defines a threshold about which unrecoverable permanent deformation occurs (deformation), marking the initiation of damage accumulation with the appearance of microcracks that occur on the periosteal surface of the bone subjected to tension; it is a measure of the bone strength; (ii) structural stiffness or bone rigidity $\left(W_{y} d_{y}\right)$, represents the slope of the elastic phase of the $W / d$ curve and is a measure of the resistance of the bone to deformation, or rigidity. It is generally proportional to the bone structural strength; and (iii) structural strength $\left(W_{f}\right)$, represents the value of the load at fracture and expresses directly the resistance of the whole bone to fracture, incorporating both the elastic and the plastic behaviors. The post-yield load was obtained by subtraction of the yield load to the failure load. The plasticlelastic ratio $\left(W_{f}-W_{y} / W_{y} \cdot 100\right)$ represents the fraction of the fracture load that is supported in plastic conditions.

\subsection{Determination of geometrical and material bone properties}

Micromorphometrical determination of the horizontal and vertical, external $(H, B)$ and internal $(h, b)$ diameters of the elliptic-crown-shaped fracture sections enabled calculations of the bone diaphyseal geometrical properties. Measurements were taken with a digital caliper with the aid of a magnifier $40 \times$. The geometric properties were determined as follows: [a] Bone length and diameters: the femur length was measured directly using a stereomicroscope (Stenu DV4 Stereo microscope Carl Zeiss Microimaging, Gottingen, Germany) with an accuracy of $\pm 100 \mu \mathrm{m}$; [b] Mid-diaphyseal cross-sectional area, CSA: CSA was calculated by applying the equation " $\pi(H B-h b) / 4$ ". Second moment of inertia of cortical bone (with reference to the anterior-posterior bending axis, $x C S M I$ ) was estimated by the equation " $\pi\left(B^{3} H-b^{3} h\right) / 64$ ". Bone material properties (apparent elastic modulus) was calculated from structural and geometric properties and not directly determined by mechanical means: "WyL ${ }^{3} / 48 d y . I x " ~(d y=$ maximal elastic deflection, $\mathrm{L}=$ distance between supports, $\mathrm{Ix}=\mathrm{xCSMI}, \mathrm{Wy}=$ load at the yielding point).

\subsection{Ashing of the specimens}

The left femur of each animal was ashed at $600^{\circ} \mathrm{C}$ in a muffle furnace for $18 \mathrm{~h}$ and the ash weight obtained. The total mineral content of the femur was derived from the weight of bone ash. The tissue degree of mineralization $(D M B)$, which expresses the percentage of mineral substance in the dried bone, was calculated as the ratio ash weight/dry bone weight.

\subsection{Statistical analysis}

Results were summarized as means \pm SEM and were considered statistically significant at the level of $P<0.05$. Comparisons between parameters were performed by the Student's t-test using GraphPad Prism Software (GraphPad Software, San Diego, CA, USA).

\section{Results}

During the $4 \mathrm{wk}$ training period, the increase in body weight in the EX group was less than the increase in body weight of the NE group. Final body weight was $364.75 \pm 9.49 \mathrm{~g}$ in the former and $423.67 \pm$ $9.29 \mathrm{~g}$ in the latter $(P=0.0402)$ (Fig. 3). The absolute weight of one of the major leg muscle, the 

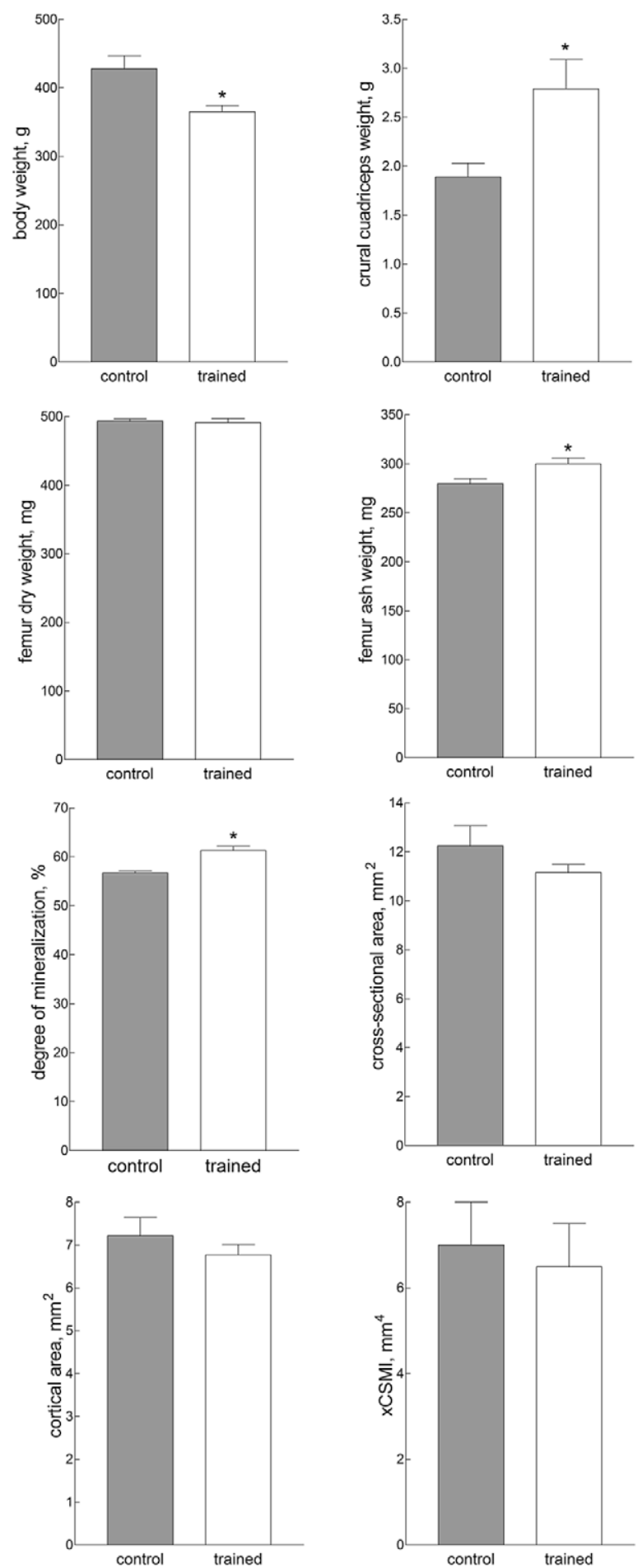

Fig. 3. Body weight, crural quadriceps weight, dry femur weight, femur ash weight, degree of mineralization, cross-sectional area, cortical area, and cross-sectional moment of inertia in control (dark bars) and trained (clear bars) of rats. Each bar represents the Mean \pm SEM of 7 animals. Asterisks on top of bars denote statistical significant differences $(P<0.05)$. 

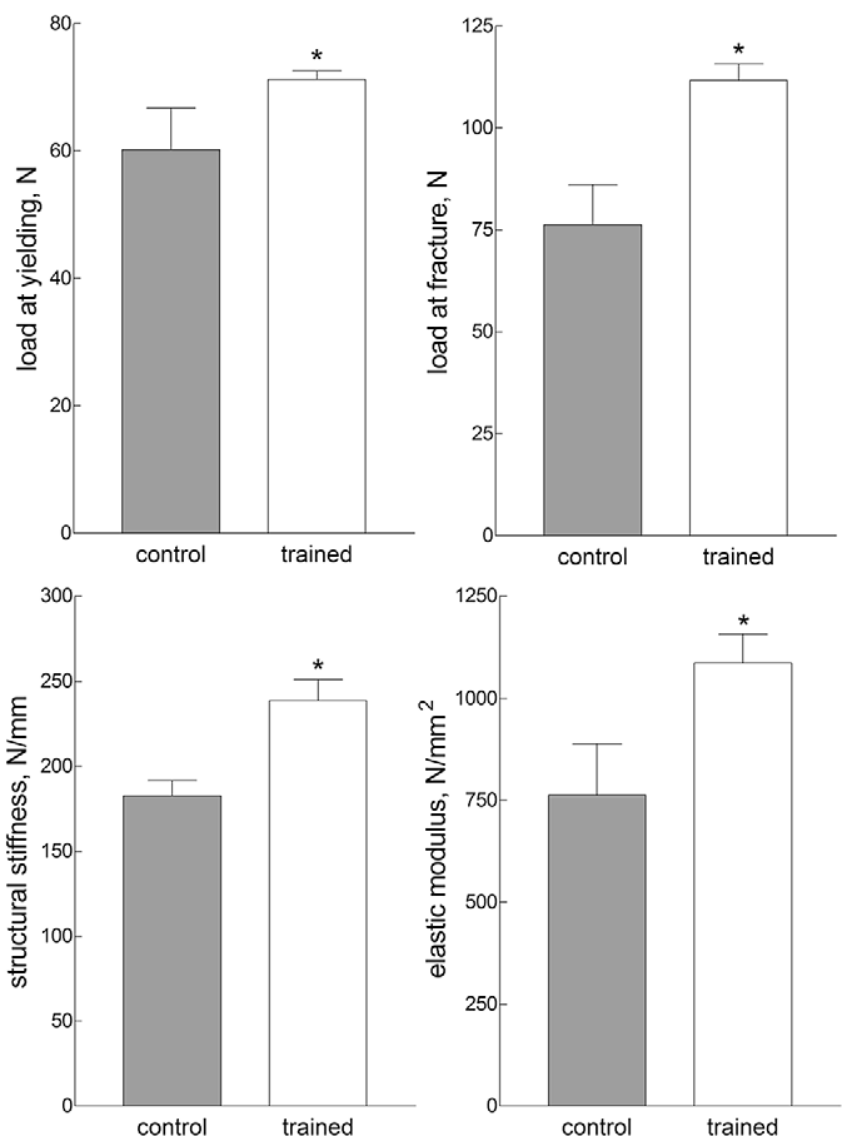

Fig. 4. Structural (load at yielding, load at fracture, and structural stiffness) and intrinsic (apparent elastic modulus) biomechanical properties in control (dark bars) and trained (clear bars) rats. Each bar represents the Mean \pm SEM of 7 animals. Asterisks on top of bars denote statistically significant differences $(P<0.05)$.

crural quadriceps, was $1.898 \pm 0.146 \mathrm{mg}$ and $2.797 \pm 0.309 \mathrm{mg}(P=0.0572)$ in the NE and EX rats, respectively, which indicates that the experimental training increased the mass of one of the associated muscles by $47.6 \%$. There was no significant difference between NE and EX rats in femur length $(32.0 \pm$ $1.3 \mathrm{~mm}$ vs. $32.3 \pm 1.2 \mathrm{~mm}$ ) nor in femoral dry weight $(493 \pm 3.3 \mathrm{mg}$ vs. $491 \pm 5.8 \mathrm{mg}$ ). Total bone ash was higher in EX than in NE rats $(301.1 \pm 6.2 \mathrm{mg}$ vs. $280.2 \pm 5.0 \mathrm{mg}$, respectively; $P=0.0335)$. The femoral degree of mineralization was also higher in the EX than in the NE rats $(61.27 \pm 0.45 \%$ vs. $56.73 \pm 0.91 \% ; P=0.0010$ ) (Fig. 3).

The results of the cross-sectional measurements performed on the breaking site of femora after the 3point bending test showed that no statistically significant differences were encountered between NE and EX rats in the cross-sectional area, cortical cross-sectional area, and cross-sectional moment of inertia (Fig. 3).

The structural mechanical properties of the femoral diaphyses are shown in Fig. 4. The load at yielding, the load at fracture, and the structural stiffness were all significantly higher in the trained than in the control rats, indicating that both the resistance to deformation under the applied load and the structural strength were improved by treatment. The apparent elastic modulus, calculated from geometrical and 
structural measured values, was $762.8 \pm 126.3 \mathrm{~N} / \mathrm{mm}^{2}$ and $1086.4 \pm 71.2 \mathrm{~N} / \mathrm{mm}^{2}(P=0.0448)$ in NE and EX rats, respectively (Fig. 4).

\section{Discussion}

Skeletal functional integrity can be assessed by structural strength tests that measure how well the whole bone can bear load [17]. During a whole bone structural test (WBSS) different types of loads can be applied to whole bones in vitro to determine both the structural stiffness (rigidity) and the structural strength (failure load). These two whole bone measurements are called structural properties and are influenced by both the material from which the structure is composed (tissue material properties) as well as the quantity (bone mass) and how and where the material is distributed (the geometric form of the tissue). Currently, there is no substitute for a WBSS to measure whole bone structural behavior; no alternative parameter has been identified that is fully indicative of strength and can serve as a surrogate measure. Bone material properties are independent of the size and shape of the bone and can be estimated by the modulus of elasticity. These properties are influenced by mineral density, collagen content, and ash fraction. In addition, factors such as collagen cross-linking, collagen fibers orientation, mineral crystal size and the microstructural organization (lamellae, osteons) also influence material behavior.

Most of the mechanical forces that act on the skeleton during physical activities are generated either through impact with the ground (i.e., gravitational or ground-reaction forces) or through skeletal muscle contractions [3]. However, conclusive evidence is lacking on which of this mechanical factors is more important to induce changes in the bone mechanical properties. Turner and Robling [18] have shown that adult female rats subjected to $16 \mathrm{wk}$ of axial loading of the right ulna showed small increases in bone mineral content (BMC) and BMD (15-17\%) but substantial increases (64-94\%) in ultimate force and energy to failure.

The present investigation was designed to estimate the structural properties of one of the weightbearing bones, the femur. Young adult rats ( 8 weeks old) were chosen because it is a period of enhanced bone deposition [19]. The animals were arranged in two groups. One of the groups (NE) was maintained in cages in which they performed the habitual low muscular activity, represented mainly by tonic contractions in order to support body weight and relatively low phasic contractions necessary to walk and get access to food and water. The other group was formed by rats (EX) that, besides performing the same types of muscular activities, were trained to run in a treadmill during $2 \mathrm{~h}$ in the morning and $2 \mathrm{~h}$ in the afternoon, at a velocity of $27 \mathrm{~m} / \mathrm{min}$ during $15 \mathrm{~d}$. During the previous 15 days, animals were preconditioned by following the same training protocol, although the velocity was increased from $0 \mathrm{~m} / \mathrm{min}$ to $27 \mathrm{~m} / \mathrm{min}$ progressively.

The structural properties of bone are regulated according to the "mechanostat theory" [6], that postulates that increasing maximal muscle force during growth or in response to increased loading will affect bone mass, size and geometry, thus adapting bone strength and stiffness to the loads acting on it. The mechanical properties of a bone depend primarily on the properties of its constituent material (material properties) and secondarily on the form that this material is distributed (geometric or architectural properties).

There are three ways to make the skeleton stronger: 1) increasing the bone mass (BMC), 2) distributing bone mass more efficiently (xCSMI), and 3 ) improving the material properties (stronger at the tissue level) (elastic modulus) (16).

As directed by the mechanostat, when a long bone, as our rat femur, is subjected to increased loads, the changes that occur are mainly, if not solely, restricted to its geometrical properties. The cross-sectional 
area (CSA) increases because of positive modeling on the periosteal surface and this response is the most important determinant with respect to the cortical thickness on bone mechanical properties, particularly for the failure load and stiffness [20]. At the same time, the endosteal surface of the bone is reabsorbed by negative modeling but at a lower rate that the positive modeling occurring at the periosteal surface. As the result of these actions, the diaphysis of the loaded long bone increases the cortical section (CSA), the thickness of the mineralized-cross-sectional area (CtCSA), and the peripherization of the mineralized tissue (xCSMI). All of these actions increase the strength of the bone and make it more resistant to fracture. The stiffness of the bone also increases, which make the bone more rigid. The structural stiffness of a given bone depends on the stiffness of the bone material tissue (intrinsic stiffness) and the geometrical properties of the bone.

In the current study, the trained animals showed a lower body weight and higher muscle weight due to exercise. However the femur length, an indirect measure of longitudinal bone growth, and its dry weight did not show any difference. The structural mechanical properties of the femoral shaft, which is mostly composed of compact bone, were enhanced by endurance training, as revealed by substantial increases in load at yielding, load at fracture, and structural stiffness (under elastic conditions). In elastic structures, all deformation energy is returned and no damage or heat is dissipated within the bone [15]. Surprisingly, the increased mechanical structural properties observed in our loaded femur were not associated with significant variations of the geometric properties of the bone. The cross-sectional area and the cortical thickness that represent the cross-sectional diameters and the amount of mineralized tissue, respectively, at the site of fracture, and the xCSMI, that shows the architectural design of the femoral shaft, were not affected by training. Thus, the main factors that make the bone stronger during loading did not apparently intervene in the genesis of the increment in the femoral structural properties in our trained rats. These findings do not agree with the rules dictated by the mechanostat and suggest that the enhanced stiffness and strength observed was the result of changes occurring in the tissue biomaterial properties. It is also possible that the mechanostat had not enough time to induce the adaptive modifications at the level of the bone geometrical properties or that they were too small to be measured with our methods. However, others have shown no geometric adaptation of weight-bearing bones in exercise trained rats in spite of increased strength and stiffness $[9,12,13,21]$.

The plastic behavior of bones depends, in addition, on the bone tissue resistance to the development and progress of cracks within the bone's structure, a property known as bone toughness. The post-yield load was more than two times higher in the EX than in the NE rats, which suggests that the increased failure load found in the former than in the latter could have been also caused by an increase in the energy developed during the irreversible deformation and that the femoral shaft could have been able to withstand greater deformation before fracture.

The intrinsic stiffness represents the rigidity of the material bone tissue, which is independent of the geometric properties of the bone. The degree of mineralization, or mineral density, has an important influence on bone strength through its effect on the intrinsic stiffness. In the current study, the degree of mineralization was $8.0 \%$ higher in the EX than in the NE rats, which would have been an important determinant of the $42.4 \%$ increase in the apparent elastic modulus observed in the former than in the latter.

The higher stiffness, failure load and post yield load were, as cited before, higher in the EX than in the NE rats. The mineralization density was also higher in the former than in the latter. These observations suggest that exercise influenced the mineralization of bone and at least partially explain bone tissue material level properties. Similar results were observed in rats with increased calcium intake [20] and points out the important role of bone mineralization on bone strength not via bone geometrical properties 
but the quality of the bone material. The amount of bone ash and the degree of mineralization are the major intrinsic determinants of bone strength at the tissue level [21].

In summary, under the exercise regimen imposed to rats in the present experiment, the femur increased significantly its structural properties, thus being more rigid and more resistant to fracture. These responses were not apparently associated with modifications of the architectural design of the femoral shaft, which did neither increase the cross-sectional nor the mineralized cortical areas nor the crosssectional moment of inertia. The findings of an increased apparent elastic modulus probably associated to an augmented mineralization density and ash content suggest that the present endurance training enhanced bone biomaterial properties acting at the tissue-level properties. The operating mechanism of this response, possibly associated to the high degree of the stimulation and the short time of application, remains as an open question. This is the reason that made us to hesitate to propose this mechanism as a rapid one to make the bone more resistant to fracture to adapt to new conditions that develop suddenly, by knowing that the architectural design of the diaphysis (cross-section and moment of inertia) needs time to develop.

\section{Conflict of interest}

The authors have no conflict of interest to report.

\section{References}

[1] D.J. DiGirolamo, D.P. Kiel and K.A. Esser, Bone and skeletal muscle: Neighbors with close ties, J Bone Min Res. 28(7) (2013), 1509. doi:10.1002/jbmr.1969.

[2] F. Rauch, D.A. Bailey, A. Baxter-Jones, R. Mirwald and R. Faulkner, The "muscle-bone unit" during the pubertal growth spurt, Bone. 34(5) (2004), 771. doi:10.1016/j.bone.2004.01.022.

[3] W.M. Kohrt, D.W. Barry and R.S. Schwartz, Muscle forces or gravidity: What predominates mechanical loading of bone?, Med Sci Sports Exerc. 41 (2009), 2050-2055. doi:10.1249/MSS.0b013e3181a8c717.

[4] C.H. Turner, Muscle-bone interactions, revisited, Bone. 27(3) (2000), 339-340. doi:10.1016/S8756-3282(00)00349-5.

[5] D.B. Burr, Muscle strength, bone mass, and age-related bone loss, J Bone Miner Res. 12 (1997), 1547. doi:10.1359/jbmr. 1997.12.10.1547.

[6] H.M. Frost, On our age-related bone loss: Insights from a new paradigm, J Bone Miner Res. 12(10) (1997), $1539-1546$. doi:10.1359/jbmr.1997.12.10.1539.

[7] H.M. Frost and E. Schônau, The "muscle-bone unit" in children and adolescents: A 2000 overview, J Pediatr Endocrinol Metab. 13(6) (2000), 571-1590. doi:10.1515/JPEM.2000.13.6.571.

[8] J. Iwamoto, C. Shimamura, T. Takeda, H. Abe, S. Ichimura, Y. Sato and Y. Toyama, Effects of treadmill exercise on bone mass, bone metabolism, and calciotropic hormones in young growing rats, J Bone Miner Metab. 22(1) (2004), $26-31$. doi:10.1007/s00774-003-0443-5.

[9] X. Chen, H. Aoki and Y. Fukui, Effect of exercise on bone strength, bone mineral density, and metal content in rat femurs, BioMed Mat Engin. 14 (2004), 53.

[10] J. Iwamoto, T. Takeda and Y. Sato, Effect of treadmill exercise on bone mass in female rats, Exp Anim. 54(1) (2005), 1-6. doi:10.1538/expanim.54.1.

[11] Y. Hagihara, S. Fukuda, S. Goto, H. Iida, M. Yamasaki and H. Moriya, Haw many days per week should rats undergo running exercise to increase BMD?, J Bone Min Metab. 23 (2005), 289-294. doi:10.1007/s00774-005-0601-z.

[12] T.H. Huang, F.L. Chang, S.C. Lin, S.H. Liu and R.S. Yan, Endurance treadmill running benefits the biomaterial quality of bone in growing male Wistar rats, J Bone Miner Metab. 26(4) (2008), 350-357. doi:10.1007/s00774-007-0831-3.

[13] T.H. Huang, J. Lewis, H.S. Lin, L.T. Kuo, S.W. Mao, Y.S. Tai et al., A methionine-restricted diet and endurance exercise decrease bone mass and extrinsic strength but increase intrinsic strength in growing male rats, $J$ Nutr. 144(5) (2014), 621630.

[14] A. Hoshi, H. Watanabe, M. Chiba and Y. Inaba, Effects of exercise at different ages on bone density and mechanical properties of femoral bone of aged mice, Tokoku J Exp Med. 1 (1998), 15. doi:10.1620/tjem.185.15. 
[15] J.L. Ferretti, G.R. Cointry, R.F. Capozza, R. Capiglioni and M.A. Chiappe, Analysis of biomechanical effects on bone and on the muscle-bone interactions in small animal models, J Musculoskel Neuron Interact. 1(3) (2001), 263-274.

[16] C.H. Turner and D.B. Burr, Basic biomechanical measurements of bone. A tutorial, Bone. 14(4) (1993), 595-608. doi:10. 1016/8756-3282(93)90081-K.

[17] M.C.H. var der Meulen, K.J. Jepsern and B. Milik, Understanding bone strength: Size isn't everything, Bone. 29(2) (2001), 101-104. doi:10.1016/S8756-3282(01)00491-4.

[18] C.H. Turner and A.G. Robling, Designing exercise regimens to increase bone strength, Exerc Sport Sci Rev. 31(1) (2003), 45-50. doi:10.1097/00003677-200301000-00009.

[19] P.H. Bonjour, G. Theintz, B. Buchs, D. Slosman and R. Rizzoli, Critical years and stages of puberty for spinal and femoral bone mass accumulation during adolescence, Clin Endocrinol Metab 73 (1991), 555-563. doi:10.1210/jcem-73-3-555.

[20] S. Viguet-Carrin, M. Hoppler, F. Membrez Scalfo, J. Vuichoud, M. Vigo, E.A. Offord and P. Ammann, Peak bone strength is influenced by calcium intake in growing rats, Bone 68 (2014), 85-91. doi:10.1016/j.bone.2014.07.029.

[21] T.H. Huang, S.C. Lin, F.L. Chang, Hsieh, S.H. Liu and R.S. Yang, Effect of different exercise modes on mineralization, structure, and biomechanical properties of growing bone, J Appl Physiol. 95(1) (2003), 300-307. doi:10.1152/ japplphysiol.01076.2002. 